\title{
Review of triple-frequency GNSS: ambiguity resolution, benefits and challenges
}

\author{
Bofeng Li
}

\begin{abstract}
Triple-frequency GNSS has been intensively studying in the past decades, especially with the open service of China's BeiDou system. In this review, we will address the ambiguity resolution, benefits gained from additional frequency signals compared to the dual-frequency GNSS signals, as well as analyse the challenges of triple-frequency GNSS for future development. We first review and compare the three carrier ambiguity resolution models of geometry-based, geometry-free, geometry-ionosphere-free (GIF). The benefits gained from triple-frequency GNSS are then comprehensively examined with respect to dual-frequency case, including the improved ambiguity resolution, extra-widelane based RTK, the augmented RTK service, the shortened PPP convergence, the improved availability and reliability. In addition, some challenges are discussed from both theoretical and practical aspects to open eyes for future research.
\end{abstract}

Keywords: Global navigation satellite systems (GNSS), Real time kinematic (RTK), Availability, Reliability, Three carrier ambiguity resolution (TCAR)

\section{Introduction}

As confirmed so far, there are at least three global GNSS systems which are broadcasting or will be upgraded to incorporate triple frequency signals, i.e., modernized GPS, Galileo and BeiDou systems. Especially since the BeiDou Inter-face Control Document was released at the end of 2012, many folks have been intensifying their efforts to the scientific and practical research of three frequency GNSS. Generally, with the additional frequency signals, it is desirable to speed up the carrierphase ambiguity resolution (AR), mitigate the various categories of error sources, reduce the communication bandwidth of transmission and so on, therefore improving both availability and reliability of the augmented RTK applications (Herandez-Pajares et al. 2003; Richert and El-Sheimy 2007; Feng and Li 2008, 2010).

Most of previous studies of making use of the additional frequency signals have concentrated on three carrier ambiguity resolution (TCAR), including the earlier contributions by Forssell et al. (1997); Vollath et al. (1998); Hatch et al. (2000); Teunissen et al. (2002); Feng and Rizos (2005); Fernández-Plazaola et al. (2004) and

Correspondence: bofeng_li@tongji.edu.cn

College of Surveying and Geo-Informatics, Tongji University, Shanghai, People's Republic of China

(c) The Author(s). 2018 Open Access This article is distributed under the terms of the Creative Commons Attribution 4.0 International License (http://creativecommons.org/licenses/by/4.0/), which permits unrestricted use, distribution, and reproduction in any medium, provided you give appropriate credit to the original author(s) and the source, provide a link to the Creative Commons license, and indicate if changes were made. the recent contributions by Cocard et al. (2008); Feng (2008); Fernández-Plazaola et al. (2008); Feng and Li (2010); Li et al. (2010a); Henkel and Günther (2012); Geng and Bock (2013); Wang and Rothacher (2013); Li et al. (2015b). A common procedure for TCAR is, given three frequencies, to identify the three best combination observables to allow for more reliable AR under the given observational conditions characterized by the magnitudes of atmospheric conditions, phase noise and orbital error etc. The selected combinations often have minimum or low ionospheric effects. The experimental results show that the extra-wide-lane (EWL) ambiguities can be reliably solved instantaneously or with very few epochs nearly without distance restriction, but the narrow-lane (NL) AR is still challenging over long baselines (Li et al. 2010a; Wang and Rothacher 2013) although it can be reliably solved over short baselines (Deng et al. 2014; Shi et al. 2013; He et al. 2014; Montenbruck et al. 2013; Odolinski et al. 2013).

Regarding the triple-frequency RTK, the studies are mainly based on the simulated data before the real data available. With the real BeiDou data, the most of studies are based on the short baselines $(<10 \mathrm{~km})$ (Shi et al. 2013; Odolinski et al. 2013; Montenbruck et al. 2013; He et al. 2014). It is therefore of greater interest to see
Springer Open 
performance over long baselines. Li et al. (2015a) systematically studied the triple-frequency long-baseline RTK with EWL and NL observations where the partial NL AR is applied. Besides, Li et al. (2017a) proposed an ERTK concept for (sub)decimetre long-baseline RTK only by using (equivalent) two EWL signals with their easier AR.

In the previous studies, we somehow always emphasize on TCAR and precise positioning. Another important benefit, the improved observation redundancy and then the reliability ( $\mathrm{Li}$ et al. 2013, 2017b), gained from additional frequency signals does not attract the enough attentions. Here the reliability is referred to as capability of observation system to resist the observation abnormality and outliers. However, to achieve the realistic reliability measures, one has to first assess the correct stochastic model of triple-frequency signals (Li 2016).

In this review, the varying triple-frequency AR models are first compared. Then, the benefits gained from the additional frequency signals are investigated comparing with the dual-frequency case. Finally, some challenges on the application of three frequency GNSS signals are discussed.

\section{Triple-frequency GNSS observation equations}

Considering the atmospheric effects, the triple-frequency double differenced (DD) observation equations read

$$
\mathrm{E}\left[\begin{array}{c}
\boldsymbol{p} \\
\boldsymbol{\phi}
\end{array}\right]=\left[\begin{array}{cccc}
\boldsymbol{e}_{3} \otimes \boldsymbol{A} & \boldsymbol{e}_{3} \otimes \boldsymbol{g} & \boldsymbol{\mu} \otimes \boldsymbol{I}_{s} & \mathbf{0} \\
\boldsymbol{e}_{3} \otimes \boldsymbol{A} & \boldsymbol{e}_{3} \otimes \boldsymbol{g} & -\boldsymbol{\mu} \otimes \boldsymbol{I}_{s} & \boldsymbol{\Lambda} \otimes \boldsymbol{I}_{s}
\end{array}\right]\left[\begin{array}{c}
\boldsymbol{x} \\
\tau \\
\boldsymbol{\iota} \\
\boldsymbol{a}
\end{array}\right]
$$

where $\boldsymbol{p}=\left[\boldsymbol{p}_{1}^{T}, \boldsymbol{p}_{2}^{T}, \boldsymbol{p}_{3}^{T}\right]^{T}$ is the code observation vector with $\boldsymbol{p}_{i}$ the observation vector of frequency $f_{i}$. $\boldsymbol{\phi}$ is the phase observation vector, having the same structure as code. $\boldsymbol{A}$ is the matrix to baseline parameter $\boldsymbol{x}$ while $\boldsymbol{g}$ is the mapping function vector to zenith tropospheric delay (ZTD) $\tau$ after correcting with standard troposphere model; $\boldsymbol{\mu}=\left[\mu_{1}, \mu_{2}, \mu_{3}\right]^{T}$ with $\mu_{j}=f_{1}^{2} / f_{j}^{2}$ is the scalar vector to DD ionospheric parameter vectorı. $I$ is the identity matrix with dimension of number of $\mathrm{DD}$ satellites. $\boldsymbol{\Lambda}=\operatorname{diag}\left(\left[\lambda_{1}, \ldots, \lambda_{f}\right]\right)$ is diagonal matrix to three frequency DD ambiguity vector $\boldsymbol{a}=\left[\boldsymbol{a}_{1}^{T}, \boldsymbol{a}_{2}^{T}, \boldsymbol{a}_{3}^{T}\right]^{T}$. $\boldsymbol{e}_{3}$ is a $(3 \times 1)$ vector with all entries of 1 . The stochastic model can be generalized as

$$
\mathrm{D}\left[\begin{array}{l}
\boldsymbol{p} \\
\phi
\end{array}\right]=\left[\begin{array}{cc}
\boldsymbol{Q}_{\boldsymbol{p}} & 0 \\
0 & \boldsymbol{Q}_{\phi}
\end{array}\right] \otimes \boldsymbol{Q}=\boldsymbol{Q}_{f} \otimes \boldsymbol{Q}
$$

where $\boldsymbol{Q}_{\boldsymbol{p}}=\operatorname{diag}\left(\left[\sigma_{p_{1}}^{2}, \sigma_{p_{2}}^{2}, \sigma_{p_{3}}^{2}\right]\right)$ and $\boldsymbol{Q}_{\boldsymbol{\phi}}=\operatorname{diag}\left(\left[\sigma_{\phi_{1}}^{2}\right.\right.$, $\left.\left.\sigma_{\phi_{2}}^{2}, \sigma_{\phi_{3}}^{2}\right]\right)$ with $\sigma_{p_{j}}^{2}$ and $\sigma_{\phi_{j}}^{2}$ the variance scalars of undifferenced code and phase on the $j$ th frequency. One of important benefits from the triple-frequency signals is to form more useful combinations. Given the combination coefficients, say, $i, j, k$ for phase and $l, m, n$ for code, the combined observation equations read

$$
\mathrm{E}\left[\begin{array}{c}
\boldsymbol{p}_{(l, m, n)} \\
\boldsymbol{\phi}_{(i, j, k)}
\end{array}\right]=\left[\begin{array}{cccc}
\boldsymbol{A} & \boldsymbol{g} & \mu_{(l, m, n)} \boldsymbol{I}_{s} & \mathbf{0} \\
\boldsymbol{A} & \boldsymbol{g} & -\mu_{(i, j, k)} \boldsymbol{I}_{s} & \lambda_{(i, j, k)} \boldsymbol{I}_{s}
\end{array}\right]\left[\begin{array}{c}
\boldsymbol{x} \\
\tau \\
\boldsymbol{\imath} \\
\boldsymbol{a}
\end{array}\right]
$$

where the combined DD code and phase observations are (Feng and Rizos 2005)

$$
\begin{aligned}
p_{(l, m, n)} & =\frac{l \cdot f_{1} \cdot p_{1}+m \cdot f_{2} \cdot p_{2}+n \cdot f_{3} \cdot p_{3}}{l \cdot f_{1}+m \cdot f_{2}+n \cdot f_{3}} \\
\phi_{(i, j, k)} & =\frac{i \cdot f_{1} \cdot \phi_{1}+j \cdot f_{2} \cdot \phi_{2}+k \cdot f_{3} \cdot \phi_{3}}{i \cdot f_{1}+j \cdot f_{2}+k \cdot f_{3}}
\end{aligned}
$$

where the combination coefficients $l, m, n$ and $i, j, k$ are all integers. For the definitions of wavelength, ambiguity, ionosphere factor in combination, one can refer to Feng (2008). The uncertainty of phase $\phi_{(i, j, k)}$ follows

$$
\sigma_{\phi_{(i, j, k)}}=\frac{\sqrt{i^{2} f_{1}^{2}+j^{2} f_{2}^{2}+k^{2} f_{3}^{2}}}{i \cdot f_{1}+j \cdot f_{2}+k \cdot f_{3}} \sigma_{\phi}
$$

In above derivation and following discussions, we further assume the unique variance, $\sigma_{\phi}^{2}$ and $\sigma_{p}^{2}$, for triplefrequency phase and code, respectively.

The different combinations can be obtained by assigning the different sets of integer coefficients, of which the wavelength will be significantly different. Generally, for the combinations with longer wavelength relative to the noise in cycle, their ambiguities are easier to be fixed. Typically, the EWL/WL ambiguities are much easier to be solved than those of NL. Here the NL means that whose wavelength is shorter than the shortest wavelength of uncombined three frequencies. However, only two of EWL/WL combinations are independent. In other words, one must solve one of NL combinations to recover all triple-frequency ambiguities (Li et al. 2015a).

\section{Model comparison of TCAR}

Three typical TCAR models are proposed, namely, geometry-based, geometry-free and geometry-ionospherefree (GIF) model, respectively. In geometry-based model, one parameterizes the DD observation equations in terms of the baseline components, while in geometry-free model, in terms of the DD receiver-satellite ranges. In the GIF model, both geometric and ionospheric terms are eliminated. Besides, to improve the model strength and meantime make use of ambiguity-fixed EWL observations, a new model is presented at last for NL AR. 


\section{Geometry-based model}

In geometry-based model, the atmospheric effects are compensated by setting up the relevant parameters. The ambiguities are solved together with the parameters, $x, \tau$ and $\iota$, based on model (3). Some studies have already determined the useful combinations for better geometrybased AR. Richert and El-Sheimy (2007) defined some useful combinations for triple-frequency GPS and Galileo. Feng (2008) identified three most useful combinations for each triple-frequency GNSS services based on the total noise level in cycles, see also (Li et al. 2010a; Li et al. 2015a). Overall, no matter what method is used, the identified useful combinations are quite similar or even equivalent. The total noise level relative to the combined wavelength in cycles, including the effects of orbital, ionospheric and tropospheric biases and phase noises, is defined as

$$
\sigma_{T C}=\sqrt{\delta_{o}^{2}+\delta_{\tau}^{2}+\mu_{(i, j, k)}^{2} \delta_{\iota}^{2}+\sigma_{\phi_{(i, j, k)}}^{2}} / \lambda_{(i, j, k)}
$$

where $\delta_{o}, \delta_{l}$, and $\delta_{\tau}$ denote the orbital, ionospheric and tropospheric bias, respectively.

Given three sets of error budgets, typically representing the short, medium and long baselines with their lengths $d$ satisfying with $d \leq 100 \mathrm{~km}, 100 \& \mathrm{lt} ; d \leq 200 \mathrm{~km}$ and $d \geq$ $200 \mathrm{~km}$, respectively (Li et al. 2010a), Table 1 presents the total noise level $\sigma_{\mathrm{TC}}$ for the useful combinations of GPS and BDS systems. For each system, the first 4 combinations are the EWL/WL, while the last two the NL. For full triple-frequency AR, one can choose two EWL/WL and one NL combinations. Apparently, the total noise level for the EWL $\phi_{(1,-6,5)}$ of GPS is about 0.160 to 0.174 cycles and $\phi_{(1,4,-5)}$ of BDS 0.136

Table 1 Total noise level $\sigma_{\mathrm{TC}}$ for geometry-based useful combinations under different given error budget in $\mathrm{cm}\left[\sigma_{\varphi}=5 \mathrm{~mm}\right]$

\begin{tabular}{lllll}
\hline system & combinations & $\delta_{l}=10$ & $\delta_{l}=20$ & $\delta_{l}=100$ \\
& & $\delta_{T}=5$ & $\delta_{T}=10$ & $\delta_{T}=15$ \\
& & $\delta_{0}=1$ & $\delta_{0}=2$ & $\delta_{0}=8$ \\
\hline GPS & $\varphi_{(0,1,-1)}$ & 0.042 & 0.067 & 0.297 \\
& $\varphi_{(1,-6,5)}$ & 0.160 & 0.163 & 0.174 \\
& $\varphi_{(1,-5,4)}$ & 0.138 & 0.154 & 0.358 \\
& $\varphi_{(1,-1,0)}$ & 0.164 & 0.322 & 1.510 \\
& $\varphi_{(4,-3,0)}$ & 0.468 & 0.913 & 2.044 \\
& $\varphi_{(4,0,-3)}$ & 0.487 & 0.951 & 1.998 \\
& $\varphi_{(0,-1,1)}$ & 0.045 & 0.074 & 0.329 \\
& $\varphi_{(1,4,-5)}$ & 0.136 & 0.138 & 0.172 \\
& $\varphi_{(1,3,-4)}$ & 0.111 & 0.122 & 0.255 \\
& $\varphi_{(1,-1,0)}$ & 0.167 & 0.330 & 1.540 \\
& $\varphi_{(4,-3,0)}$ & 0.466 & 0.909 & 1.618 \\
& $\varphi_{(5,-4,0)}$ & 0.537 & 1.040 & 1.919 \\
\hline
\end{tabular}

to 0.172 cycles even with the large orbital and atmospheric biases over long baselines. It reveals that over long baselines the instantaneous EWL AR is achievable while it is difficult for NL AR with geometrybased model.

\section{Geometry-free model}

In the geometry-free model, the geometric terms are directly estimated instead of their linearization with respect to baseline and ZTD parameters. The mathematic model reads

$$
\mathrm{E}\left[\begin{array}{c}
\boldsymbol{p}_{(l, m, n)} \\
\boldsymbol{\phi}_{(i, j, k)}
\end{array}\right]=\left[\begin{array}{ccc}
\boldsymbol{I}_{s} & \mu_{(l, m, n)} \boldsymbol{I}_{s} & 0 \\
\boldsymbol{I}_{s} & -\mu_{(i, j, k)} \boldsymbol{I}_{s} & \lambda_{(i, j, k)} \boldsymbol{I}_{s}
\end{array}\right]\left[\begin{array}{c}
\boldsymbol{\varrho} \\
\boldsymbol{\iota} \\
\boldsymbol{a}_{(i, j, k)}
\end{array}\right]
$$

Obviously, this model is rank-deficient since the column vectors are dependent. Thus, the minimum constraints (datum) should be applied, for instance, by fixing ionospheric parameters. As a result, the float solution of ambiguities is (Feng and Rizos 2005; Li et al. 2010a)

$$
\hat{\boldsymbol{a}}_{(i, j, k)}=\frac{\boldsymbol{p}_{(l, m, n)}-\boldsymbol{\phi}_{(i, j, k)}}{\lambda_{(i, j, k)}}
$$

with its variance matrix

$$
\boldsymbol{Q}_{\hat{\boldsymbol{a}}_{(i, j, k)}}=\frac{\sigma_{p_{(l, m, n)}^{2}}^{2}+\sigma_{\boldsymbol{\phi}_{(i, j, k)}}^{2}}{\lambda_{(i, j, k)}^{2}} \boldsymbol{Q}=\left(\sigma_{\hat{a}_{(i, j, k)}}^{\mathrm{SE}}\right)^{2} \boldsymbol{Q}
$$

where $\sigma_{\hat{a}_{(i, j)}}^{\mathrm{SE}}$ denotes the formal STD of single-epoch float ambiguity estimate. Such estimated float solution could be biased due to the fixed ionospheric delays. The bias is

$$
\boldsymbol{\delta}_{\hat{\boldsymbol{a}}_{(i, j, k)}}=\frac{\mu_{(l, m, n)}+\mu_{(i, j, k)}}{\lambda_{(i, j, k)}} \boldsymbol{\iota}
$$

In this case, one can select the useful combinations based on the bias-affected success rate of integer rounding (Teunissen 2001). To give a success rate, for instance, 99.9\%. The combination schemes, $\phi_{(i, j, k)}$ along with $p_{(l, m}$, ${ }_{n \text { ) }}$ are selected. If the coefficients of ionospheric delays on phase and code satisfies with $\mu_{(l, m, n)}+\mu_{(i, j, k)}=0$, the unbiased float ambiguity solution can be computed. To use the bias-affected or unbiased model depends on the balance of bias and ambiguity precision. The reason is that with the unbiased model, the biases are explicitly modelled (parameterized) and thus the model strength is enhanced and the ambiguity precision will be improved as compared to the solution obtained with the biased model. If the AR performance with the biased model is better than with the unbiased model, i.e., the bias impact on the success rate is less than that on the ambiguity precision, it would allow for faster AR using the biased model (Verhagen et al. 2012; Li et al. 2014b). 
Table 2 The useful combination schemes for GPS and BDS EWL AR based on the geometry-free models, for which the single-epoch success rates are computed. In computation, we take $\sigma_{\varphi}=5 \mathrm{~mm}, \sigma_{p}=0.5 \mathrm{~m}$ and $\iota=0.3 \mathrm{~m}$

\begin{tabular}{|c|c|c|c|c|c|c|}
\hline system & combination & schemes & $\lambda_{(i, j, k)}[\mathrm{m}]$ & $\mu_{(i, j, k)}+\mu_{(l, m, n)}$ & $\sigma_{\hat{a}_{(0, j, k)}^{S E}}$ [cycle] & $\mathrm{P}_{\mathrm{s}}[\%]$ \\
\hline \multirow[t]{6}{*}{ GPS } & $\varphi_{(0,1,-1)}$ & $p_{(0,1,1)}$ & 5.861 & 0 & 0.067 & 100 \\
\hline & $\varphi_{(1,0,-1)}$ & $p_{(1,0,1)}$ & 0.751 & 0 & 0.477 & 100 \\
\hline & $\varphi_{(1,-6,5)}$ & $p_{(1,1,1)}$ & 3.256 & 1.360 & 0.183 & 98.01 \\
\hline & $\varphi_{(1,-6,5)}$ & $p_{(1,1,0)}$ & 3.256 & 1.209 & 0.193 & 97.86 \\
\hline & $\varphi_{(1,-5,4)}$ & $p_{(1,1,1)}$ & 2.093 & 0.773 & 0.192 & 97.97 \\
\hline & $\varphi_{(1,-5,4)}$ & $p_{(1,1,0)}$ & 2.093 & 0.622 & 0.215 & 97.50 \\
\hline \multirow[t]{6}{*}{ BDS } & $\varphi_{(0,1,-1)}$ & $p_{(0,1,1)}$ & 4.884 & 0 & 0.078 & 100 \\
\hline & $\varphi_{(1,0,-1)}$ & $p_{(1,0,1)}$ & 1.025 & 0 & 0.349 & 84.86 \\
\hline & $\varphi_{(1,4,-5)}$ & $p_{(1,1,1)}$ & 6.371 & 2.015 & 0.143 & 99.77 \\
\hline & $\varphi_{(1,4,-5)}$ & $p_{(1,1,0)}$ & 6.371 & 1.945 & 0.145 & 99.73 \\
\hline & $\varphi_{(1,3,-4)}$ & $p_{(1,1,1)}$ & 2.765 & 0.745 & 0.150 & 99.73 \\
\hline & $\varphi_{(1,3,-4)}$ & $p_{(1,1,0)}$ & 2.765 & 0.675 & 0.168 & 99.42 \\
\hline
\end{tabular}

We select several sets of combinations with unbiased or biased models. The result is presented in Table 2, where the single-epoch success rates are given as well. In general, all EWL combinations have fast AR with very high success rate. For GPS, the combinations, $\phi_{(0,1,-1)}$ with $p_{(0,1,1)}$, and $\phi_{(1,-6,5)}$ with $p_{(1,1,1)}$, are the best schemes with nearly $100 \%$ success rate; while for $\mathrm{BDS}, \phi_{(0,1,-1)}$ with $p_{(0,1,1)}$ and $\phi_{(1,4,-5)}$ with $p_{(1,1,1)}$ are the best choice. To intuitively show the ease of EWL AR, an example from real triple-frequency BDS data on a $50 \mathrm{~km}$ baseline is conducted. The fraction histogram of float ambiguities to their nearest integers is illustrated in Fig. 1 for EWL $\hat{a}_{(0,-1,1)}$ and $\hat{a}_{(1,4,-5)}$. Obviously, most of float ambiguities are very close to integer with fractions smaller than 0.3 cycles.

Compared to geometry-based model, the geometry-free model has weaker model strength and then the degraded AR performance. However, due to the extreme long wavelength of EWL signals and much simpler implementation, it is often preferable to use geometry-free model for EWL AR.

\section{GIF model for NL AR}

The studies above reveal that the EWL AR is rather easy in either geometry-free or geometry-based model, whereas the NL AR is still challenging due to their short
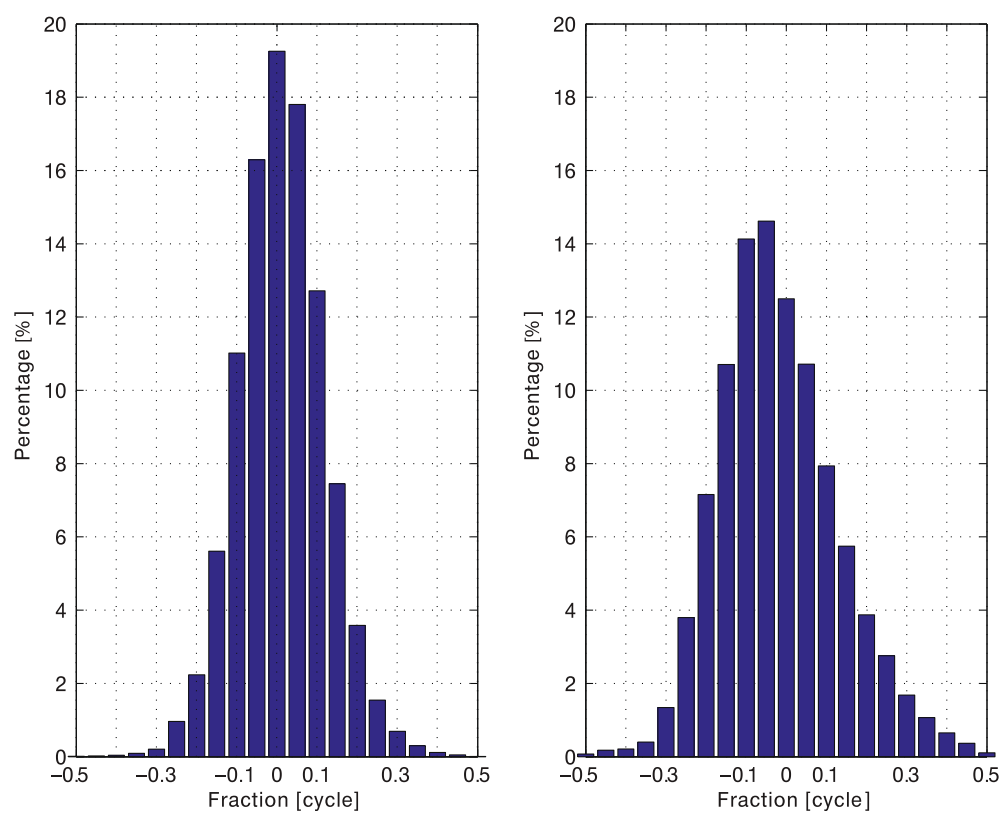

Fig. 1 Histogram of fractions of float EWL ambiguities $\hat{a}_{(0,-1,1)}$ and $\hat{a}_{(1,4,-5)}$ to their nearest integers for a $50 \mathrm{~km}$ baseline of BDS system 
wavelengths. Once two EWL/WL ambiguities are fixed, their observables can be deemed as virtual code observables except their higher accuracies than real code. One can then use these two ambiguity-fixed EWL observations, $\check{\phi}_{(l, m, n)}$ and $\check{\phi}_{(p, q, r)}$, and one NL observation, $\phi_{(i, j}$, ${ }_{k}$ ), to form a GIF combination. Then the NL ambiguity can be estimated by (Li et al. 2010a)

$$
\hat{a}_{(i, j, k)}=\frac{b_{1} \check{\phi}_{(l, m, n)}+b_{2} \check{\phi}_{(p, q, r)}-\phi_{(i, j, k)}}{\lambda_{(i, j, k)}}
$$

Following the definition of GIF model, the combination coefficients are determined by

$$
\begin{aligned}
& b_{1}=\frac{\mu_{(i, j, k)}-\mu_{(p, q, r)}}{\mu_{(l . m . n)}-\mu_{(p, q, r)}} \\
& b_{2}=1-b_{1}
\end{aligned}
$$

For arbitrary choices of EWL/WL and NL observables of GPS and BDS, the STD of NL ambiguity estimate is derived as

$$
\sigma_{\hat{a}_{(i, j, k)}}= \begin{cases}1013.5 \sigma_{\phi} & \text { GPS } \\ 1059.8 \sigma_{\phi} & \text { BDS }\end{cases}
$$

Obviously, the AR performance in GIF model is exactly equivalent for all schemes. Note the effects of geometric and ionospheric biases are totally removed for all schemes in GIF model. It is therefore promising for long baselines. With real triple-frequency data, this model has attracted more attentions. The initial results showed that it was somehow affected by some unexpected abnormality although this degradation is lamely attributed to the multipath (Wang and Rothacher 2013).

\section{Geometry-based NL AR with ambiguity-fixed EWLs}

As shown above, the geometry-based and geometry-free model can realize the EWL/WL AR with high singleepoch success rate, while they are still challenging for NL AR. The GIF model has weakest model strength for NL although it can eliminate all systematic errors. To enhance the model strength and also properly compensating the systematic errors for NL AR, instead of GIF model, we present a geometry-based model formed by code, two ambiguity-fixed EWL and L1 phase observations ( $\mathrm{Li}$ et al. 2015a)

$$
\mathrm{E}\left[\begin{array}{c}
\boldsymbol{p} \\
\boldsymbol{\phi}_{(l, m, n)} \\
\boldsymbol{\phi}_{(p, q, r)}^{\mathbf{v}} \\
\boldsymbol{\phi}_{(i, j, k)}
\end{array}\right]=\left[\begin{array}{cccc}
\boldsymbol{e}_{3} \otimes \boldsymbol{A} & \boldsymbol{e}_{3} \otimes \boldsymbol{g} & \boldsymbol{\mu} \otimes \boldsymbol{I}_{s} & \mathbf{0} \\
\boldsymbol{A} & \boldsymbol{g} & -\mu_{(l . m . n)} \boldsymbol{I}_{s} & \mathbf{0} \\
\boldsymbol{A} & \boldsymbol{g} & -\mu_{(p, q, q, r} \boldsymbol{I}_{s} & \mathbf{0} \\
\boldsymbol{A} & \boldsymbol{g} & -\boldsymbol{I}_{s} & \lambda_{1} \boldsymbol{I}_{s}
\end{array}\right]\left[\begin{array}{c}
\boldsymbol{x} \\
\tau \\
\boldsymbol{t} \\
\boldsymbol{a}
\end{array}\right]
$$

In this model, both ionospheric and tropospheric biases are absorbed by setting their associated parameters instead of eliminating in GIF model. As a result, one can impose some constraints on those parameters to enhance the model strength. Note now two EWL and NL phase observations are correlated and their correlations should be captured in the stochastic model. In addition, the filtering method can be employed by further imposing the time-varying constraints on ionospheric and tropospheric constraints (Li et al. 2015a, 2017a).

\section{Similarity and differences of TCAR from LAMBDA}

The LAMBDA method is currently the most popular AR method in GNSS community due to its efficient implementation with decorrelation technique (Teunissen 1995). Different from TCAR with pre-set combinations, LAMBDA can automatically and optimally work out such combinations (Teunissen et al. 2002). These combinations could be more complicated since it makes full use of correlation information of ambiguity variance matrix and decorrelates the ambiguities as much as possible. However, when using LAMBDA method, one needs to first select out the subset of ambiguities that can be reliably fixed, since it is usually impossible to always fix full set of ambiguities. This task is indeed troublesome in real applications. In the combination based TCAR, one commonly gets used to fixing the ambiguity individually although it loses a lot of information and is not encouraged.

The essence of LAMBDA is the decorrelation technique. We compare the combination-based and decorrelation-based TCAR identifying their similarities and differences as follows:

1. The Z-transformation matrix is automatically generated in the decorrelation process, but the combinations are pre-determined based on a certain objective.

2. The decorrelation works on the ambiguity variance matrix to make it minimally correlated, while the combination directly on the measurement domain to reduce or even eliminate effects of some biases so as to derive the minimal total noise level in cycles.

3. The combination can, to a certain extent, realize the decorrelation purpose. But this decorrelation works only between-frequency ambiguities of one pair DD satellites. However, LAMBDA decorrelation works on the ambiguities between inter-frequencies, inter-satellites and even inter-GNSS systems if available. Therefore, the multiple frequency GNSS system is necessary for combination TCAR, but LAMBDA can work on any GNSS scenarios.

4. With combination based TCAR, it is somehow used to solving the ambiguity individually, especially for EWL AR. As a consequence, the AR of one pair DD satellites cannot be affected by the abnormality of the other DD satellites. However, the decorrelation based LAMBDA method would solve the transformed 
ambiguity as a combination of several or all DD satellites. Once there is abnormality in one DD ambiguity used in the ambiguity combination, the transformed ambiguity will not be correctly solved anymore.

5. The LAMBDA method starts from the original observation Eq. (1) where all observation contents are fully used; whereas in the combination based TCAR, the observation content is more or less lost. Considering the model strength and the simplicity of combination based TCAR, we therefore suggest to use combination based TCAR for EWL AR, while the LAMBDA method for NL AR. Of course, one can first use the pre-set combination to transform the ambiguities and then further apply LAMBDA method. Such processing may speed up the decorrelation procedure.

\section{Benefits of triple-frequency GNSS Improved ambiguity resolution}

It is understandable that the additional frequency signals can improve AR with respect to dual-frequency case. The success rate comparison between dual- and triplefrequency is intuitively illustrated in Fig. 2 based on the simulation. The simulation is conducted on a $50 \mathrm{~km}$ with GPS constellation based on the geometry-based ionosphere-weighted model, for which one can refer to (Li and Teunissen, 2014). In the computations, we take the varying STD of undifference ionospheric constraint as $\sigma_{\iota}=5 \mathrm{~cm}, 10 \mathrm{~cm}, 15 \mathrm{~cm}$ and $\infty$. For $\sigma_{\iota}=\infty$, it is actually the ionosphere-float model. The success rates are computed for number of epochs from 1 to 5 .

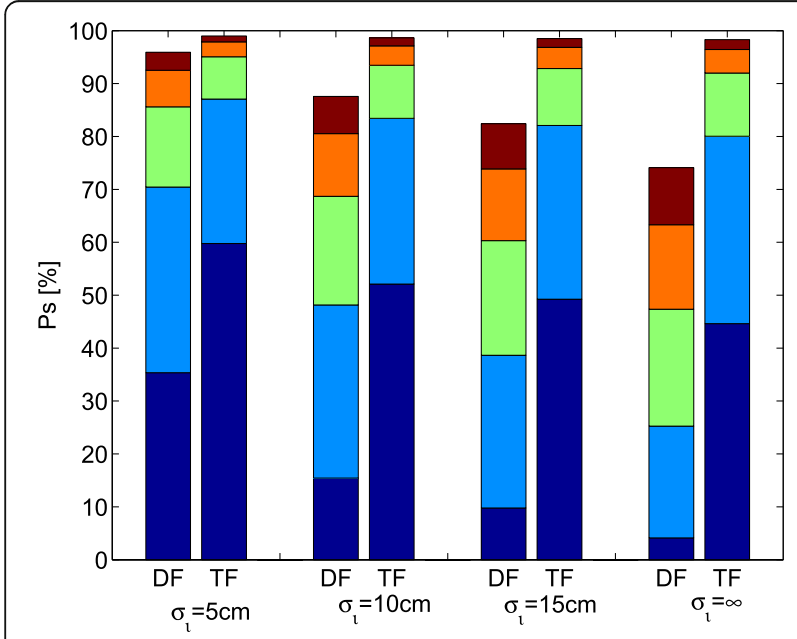

Fig. 2 The success rate of AR in the geometry-based ionosphereweighted model with different number of epochs and different variance of ionospheric constraints. The different colors in one bar indicate the accumulated success rate gained by number of epochs from 1 to 5
The result reveals that (1) the success rates of dualfrequency are significantly improved by triple-frequency and the improvement becomes more remarkable when the ionospheric constraint becomes weaker; (2) since triple-frequency geometry-based model is already very strong, the success rates of different ionospheric constraints are very similar. It is however not the case for the dual-frequency, its success rate becomes smaller for the weaker ionospheric constraint; (3) it is noted that the triple-frequency success rate is still very large in case of ionosphere-float model. This result is very promising, meaning that the AR over long baselines will be applicable with triple-frequency signals. For more information about the improved AR, one can refer to Teunissen et al. (2002); Geng and Bock (2013); He et al. (2014); Li and Teunissen (2014).

\section{ERTK with triple-frequency EWL observations}

As mentioned previously, the superiority of triplefrequency GNSS signals is to form more useful combinations, of which the EWL combinations are the most useful for instantaneous AR, with very high success rates over long baselines. However, for the long term, we start with the centimeter RTK solutions after all of the carrier ambiguities have been fixed, although this process may take many minutes. During this process, the ambiguityfixed EWL observations serve as the role of code. Actually the ambiguity-fixed EWL observations have higher precision than actual code and are thus expected to obtain a better RTK solution directly.

To make full use of the benefit of instantaneous EWL AR, Li et al. (2017a) proposed an ERTK concept where the $10 \mathrm{~cm}$ level RTK solutions can be instantaneous obtained by using the ambiguity-fixed EWL observations without NL AR. Such accurate results are very promising and already satisfy many applications without complicated NL AR. For more information about varying ERTK models, one can refer to Li et al. (2017a).

\section{Augmented GNSS RTK service}

The dual-frequency based RTK application is mainly limited typically to not longer than $20 \mathrm{~km}$. The reason is that the fast and reliable AR becomes more and more difficult with increased inter-receiver distance due to the distance-dependent atmospheric effects. If the long baseline is involved, these effects have to been carefully modelled usually by setting up the relevant parameters. As a result, the model strength will be very weak and the AR performance degrades.

With introducing the third frequency signals, the model strength can be significantly improved so that the fast and reliable AR becomes easier even over long baselines. Furthermore, the enhanced model allows us to 
careful modelling the distance-dependent systematic biases to realize the precise RTK applications over long baselines. For the single-baseline RTK, the initial studies have shown that with triple-frequency GNSS, the service distance can be extended to about $100 \mathrm{~km}$ with comparable performance to current short distance RTK with dual frequency. It means that in the medium city of China, like Shanghai, we can provide the RTK service with only one reference station (Feng and Li 2010). Analogously, for the network RTK, the distance of reference stations is advised to be roughly doubled with hexagon type of reference deployment. Such network deployment can save huge money in equipment installation and on-going maintenance (Feng and Li 2008).

\section{Shortened convergence for PPP}

Precise point positioning (PPP) has been intensively studying in past decades thanks to its convenient operation without need of reference station like in RTK (Zumbeger et al. 1997). The initial PPP was limited to the postprocessing and ambiguity-float estimation since the real-time satellite clock products are not available and the integer property of ambiguity is lost in undifference mode. By employing the network stations, the undifference phase biases and the clock corrections are estimated for ambiguity-resolved and real-time PPP. Moreover, when the network is sufficiently small, the atmospheric corrections can also be generated for augmented PPP to realize the comparable performance to network RTK (Ge et al. 2008; Collins et al. 2008; Laurichesse et al. 2008; Li et al. 2011b).

Compared to the traditional dual-frequency PPP, the studies have numerically demonstrated that the triplefrequency PPP can shorten the convergence time while the reduction of positioning errors is marginal (Geng and Bock 2013; Guo et al. 2016; Deo and El-Mowafy 2016). Note more inter-frequency biases must be introduced in the triple-frequency PPP at both satellite and receiver ends. All these biases must be carefully handled; otherwise one cannot obtain the desirable PPP solutions (Gu et al. 2015; Guo et al. 2015; Li et al. 2016). Our idea is to set up as few bias parameters as possible based on the stability analysis of these biases in order to achieve the mode strength as strong as possible.

\section{Improved availability of precise positioning}

The precise GNSS application usually needs the phase AR that takes the different time depending on the application and observation scenarios. In general, the less time cost for AR, the earlier the user can achieve the precise positioning and then the higher availability. In real applications, one cannot always fix all ambiguities due to their different precisions and one does not necessarily to fix all ambiguities in sense of precision demand. Therefore, only part of ambiguities can be reliably fixed with sufficiently large success rate ( $\mathrm{Li}$ and Teunissen 2014; Li et al. 2014a).

To demonstrate how triple-frequency improves the availability of precise positioning, we conduct the following simulations based on the geometry-based ionosphere-weighted model. Given the success rate threshold $99.9 \%$, we can then fix a subset of ambiguities for which the success rate is larger than this threshold. With accumulating more epochs, the model strength is enhanced so that more ambiguities can be gradually fixed and the positioning precision is accordingly improved. Given a user-defined positioning precision criterion, we can then compute the number of epochs at least needed to achieve this precision. The fewer the number of epochs needed, the earlier the user can start to use the obtained positioning solutions. Figure 3 shows the mean number of epochs needed to achieve the different user-defined precisions over $24 \mathrm{~h}$ for different baseline lengths specified by the STD of ionospheric constraint. The results indicate that both the lower precision demand and the smaller STD of (stronger)
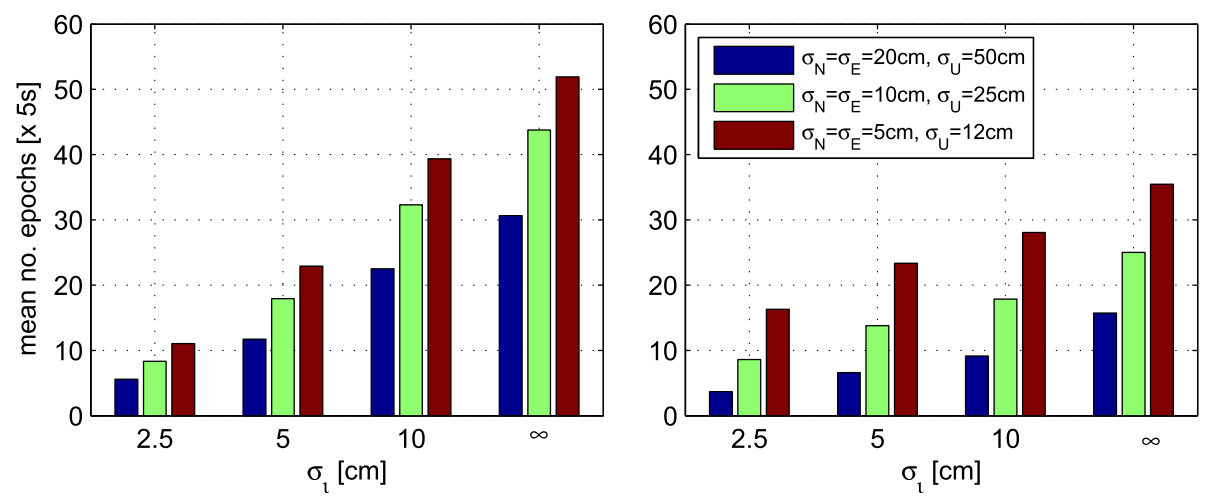

Fig. 3 Mean number of epochs used for achieving the different user-demand positioning precisions as function of the STD of ZD ionospheric constraint. The left subplot is for dual-frequency case, while the right for triple-frequency case 
ionospheric constraint will need the fewer number of epochs. The importance is that for all scenarios with different ionospheric constraints and different precision demands, the much fewer number of epochs is needed for triple-frequency than that of dual-frequency. In other words, compared with dual-frequency, triple-frequency can achieve the same precise solution with less time, and therefore improve the avail-ability of precise solutions.

Another example is presented to further show the availability improvement by triple-frequency based on the pseudorange navigation. In real situation, we make decision of accepting or rejecting navigation solution usually based on its derived variance matrix ( $\mathrm{Li}$ et al. 2013). Since the full variance matrix is relatively complicated, one then often uses one type of precision that is a function of the variance matrix, like error ellipse, mean square positional error (MSPE), instead of variance matrix itself. The MSPE is an easy-to-computed statistic defined as (Leick, 2004)

$$
\hat{\sigma}_{\mathrm{MSPE}}=\sqrt{\hat{\sigma}_{N}^{2}+\hat{\sigma}_{E}^{2}}
$$

where $\hat{\sigma}_{N}$ and $\hat{\sigma}_{E}$ are the STDs of computed north and east components. Given a user-defined threshold $\sigma_{\mathrm{MSPE} ; 0}$, the solution is accepted if its MSPE smaller than $\sigma_{\mathrm{MSPE} ; 0}$. Then the availability is defined as the percentage of accepted solutions out of all solutions. Figure 4 presents the availabilities as a function of $\sigma_{\mathrm{MSPE} ; 0}$ with different cut-off elevations. The results show that for all scenarios the availabilities are significantly improved by triple-frequency. Especially for $\sigma_{\mathrm{MSPE} ; 0}=1 \mathrm{~m}$, the dualfrequency availabilities are nearly zero but they are improved to about $20 \%$ by triple frequency.

\section{Improved reliability}

In GNSS community, we are used to pursing the improvement of AR and precise positioning. The benefit of improved reliability from triple-frequency is somehow ignored. In fact, with introducing the third frequency signals, one of important benefits is to increase the observation redundancy significantly and then improve the capability of observation system to resist the abnormality, for instance, the outliers.

Again with a pseudorange based navigation system as example, besides the high availability, a good navigation system should have the small decision error for any given criterion, on which the availability is based (Li et al. 2013). Although we have shown the higher availability of three frequency navigation system in Fig. 4, it may be not applicable if its associated decision error is large. The decision error is defined as such that the solution with large error is wrongly accepted. The decision error is measured by probability of making wrong decision. It is the frequency of wrongly accepted solutions out of total accepted solutions

$$
P_{\text {error }}=\frac{\# \text { wrongly accepted solutions }}{\text { \#accepted solutions }}
$$

Figure 5 shows the probabilities of decision errors with respect to the results in Fig. 4. The result of triplefrequency is smaller than, or at least comparable with, that of dual-frequency. Therefore, we can ensure that triplefrequency navigation has superior performance than dualfrequency case with higher availability and reliability.

\section{Discussions on some challenges}

Some challenges are discussed and the research outlooks for triple-frequency GNSS are provided in this section.

\section{Tropospheric modelling for precise RTK}

With triple-frequency GNSS, we are more interested in the precise positioning over long baselines. In such case, the ionospheric delays are basically eliminated via between-frequency combination. Regarding tropospheric delays, they can never be reduced using multiple frequencies. Their effects on the position solutions are positively proportional to the base-to-rover distance,
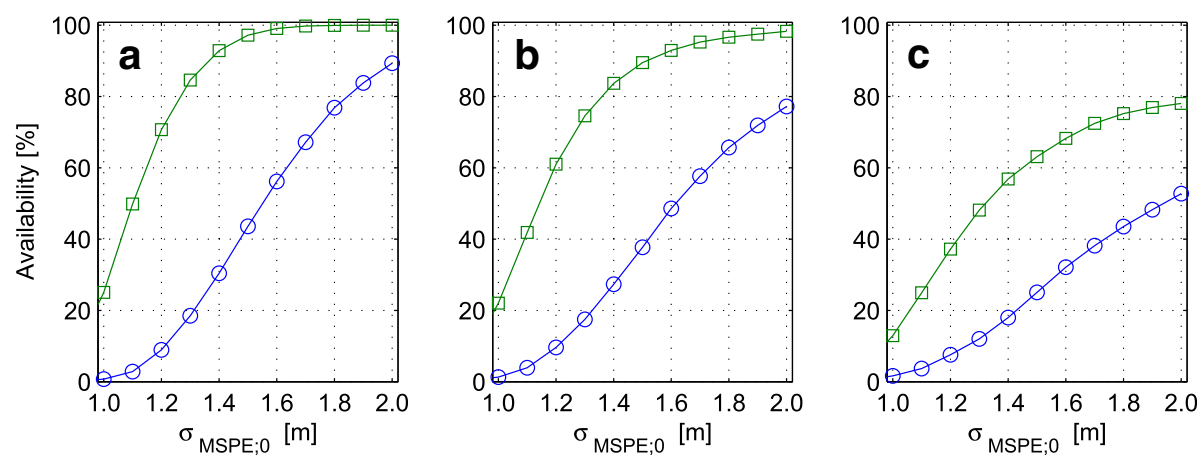

Fig. 4 The availabilities of navigation solutions as a function of user-defined MSPE $\left(\sigma_{\mathrm{MSPE}: 0}\right)$ with the different cut-off elevations, $10^{\circ}, 20^{\circ}$ and $30^{\circ}$ in subplots from (a) to (c). In each subplot, the line with squares denotes the three frequency result while the line with circles the dual-frequency result 

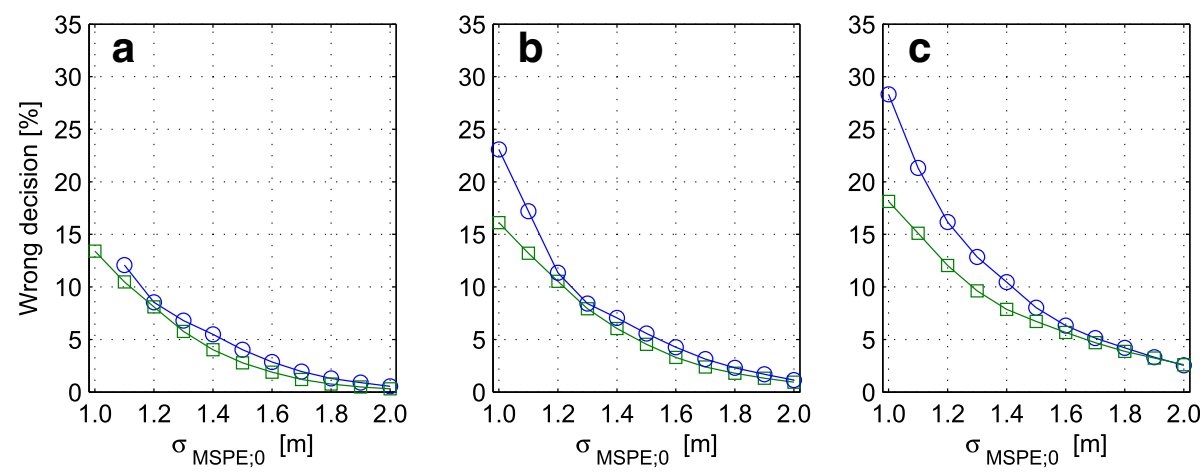

Fig. 5 The probabilities of decision errors as a function of user-defined MSPE $\left(\sigma_{\mathrm{MSPE} ; 0}\right)$ under the different cut-off elevations, $10^{\circ}, 20^{\circ}$ and $30^{\circ}$ from (a) to (c). In each subplot, the line with squares denotes the three frequency result while the line with circles the dual-frequency result

usually a few centimeter errors for the baseline of a few tens of kilometers (Dai et al. 2007). A common method is to set up a zenith tropospheric delay (ZTD) parameter with mapping function to absorb the tropospheric effects. The accuracy of such tropospheric modelling is not sufficient for precise RTK. Furthermore, the ZTD is strong correlated with the height such that the model cannot be stably solved unless a unique ZTD is assumed for a period of observations (Dodson et al. 1996; Li et al. 2010b). Such ZTD estimate cannot effectively reflect the real-time variation of troposphere environment. Therefore, how to precisely model the tropospheric biases is very critical and challenging for long baseline precise RTK with triple-frequency GNSS signals.

\section{Subset selection for partial NL AR}

In real applications, the ambiguities in the unknown ambiguity vector differ from the tracking durations, the observation geometry and the atmospheric/multipath effects. Therefore, the ambiguities generally cannot be fixed simultaneously ( $\mathrm{Li}$ et al., 2014). However, it is usually possible to fix a subset of ambiguities with high confidence. For instance, as presented above, the EWL/ WL ambiguities can be always very easily fixed though the NL AR is difficult. In fact, we do not necessarily fix all ambiguities in sense of improving baseline precision. Only the ambiguities that are sufficiently correlated with the baseline components can be used to improve baseline solution.

Considering the difficulty of NL AR, we would prefer the partial ambiguity resolution (PAR) with fixing subset of ambiguities instead of full AR. Fixing a subset of ambiguities enables the improvement of baseline solution. For some case, the baseline precision with PAR can be improved even to satisfy with the userdemand precision. Let the ambiguity vector structured as $\hat{\boldsymbol{a}}=\left[\hat{\boldsymbol{a}}_{1}^{T}, \hat{\boldsymbol{a}}_{2}^{T}\right]^{T}$ and $\boldsymbol{Q}_{\hat{\boldsymbol{a}}_{2} \hat{\boldsymbol{a}}_{2}}$ the variance matrix of $\hat{\boldsymbol{a}}_{2}$, once the subset of ambiguities $\hat{\boldsymbol{a}}_{2}$ is fixed to $\boldsymbol{a}_{2}$, the baseline solution can be updated as

$$
\begin{aligned}
& \check{\boldsymbol{b}}=\hat{\boldsymbol{b}}+\boldsymbol{Q}_{\hat{\boldsymbol{b}} \hat{a}_{2}} \boldsymbol{Q}_{\hat{a}_{2} \hat{a}_{2}}^{-1}\left(\check{\boldsymbol{a}}_{2}-\hat{\boldsymbol{a}}_{2}\right) \\
& \boldsymbol{Q}_{\check{b} \check{b}}=\boldsymbol{Q}_{\hat{\boldsymbol{b}} \hat{b}}-\boldsymbol{Q}_{\hat{\boldsymbol{b}} \hat{\boldsymbol{a}}_{2}} \boldsymbol{Q}_{\hat{\boldsymbol{a}}_{2} \hat{a}_{2}}^{-1} \boldsymbol{Q}_{\hat{a}_{2} \hat{b}}
\end{aligned}
$$

where $\boldsymbol{b}^{\nu}$ and $\hat{\boldsymbol{b}}$ are the fixed and float baseline solutions with the corresponding variance matrices, $\boldsymbol{Q}_{\dot{b} \dot{b}}$ and $\boldsymbol{Q}_{\hat{b} \hat{b}}$. $Q$ $\hat{\boldsymbol{b}} \hat{\boldsymbol{a}}_{2}$ denotes the covariance matrix of $\hat{\boldsymbol{b}}$ and $\hat{\boldsymbol{a}}_{2}$. Obviously, $\boldsymbol{Q}_{\dot{b}} \dot{b}<Q_{\hat{b} \hat{b}}$ indicates that the baseline precision is improved with fixed subset of ambiguities. Now an open problem is remained for how to reasonably choose the subset of ambiguities for PAR with both complicated real observation effects and observation geometry taking into account.

\section{Efficient stochastic modelling of triple-frequency signals}

The stochastic model is applied to describe the precision and correlation of observations. It is rather important in GNSS for reliable AR, positioning and quality control. Therefore, in past a few years, the significant research efforts have been received for refining the GNSS stochastic models (Wang et al. 1998; Tiberius and Kenselaar 2000; Li et al., 2008).

In Li (2016), the triple-frequency BeiDou stochastic model is systematically analysed by using variance component estimation. The results indicated the complexity of stochastic characteristics of triple-frequency signals especially for three types of orbiting satellites of BDS system. Hence, how to efficiently recover the precise stochastic model for (near) real-time application is very important.

As a case study shown in $\mathrm{Li}$ et al. (2011), the variance and covariance unknowns are estimated for all DD observations to recover the precise stochastic model. In such case, say $n$ DD satellites, there will be $n \times(2 n+1)$ unknowns to be estimated for dual-frequency signals. With three frequency signals, the unknown components will be increased to $3 n \times(3 n+1) / 2$. It means that $\left(5 n^{2}+n\right) / 2$ 
more unknowns need to be estimated with one frequency additional signals. Such number of unknowns is unacceptable for real-time GNSS applications. For instance, 65 more parameters are introduced for $n=5$. Therefore, how to refine the stochastic model and with which to reduce the number of unknowns in real GNSS applications needs more research attentions.

\section{Funding}

This manuscript is fully supported by National Key Research and Development Program of China (2016YFB0501802).

\section{Availability of data and materials}

Not applicable

\section{Author's contributions}

This is a single authored paper. I made $100 \%$ contribution to the paper.

\section{Competing interests}

I declare that I have no competing interests.

\section{Publisher's Note}

Springer Nature remains neutral with regard to jurisdictional claims in published maps and institutional affiliations.

Received: 4 October 2017 Accepted: 23 January 2018

Published online: 12 February 2018

\section{References}

Cocard M, Bourgon S, Kamali O, Collins P (2008) A systematic investigation of optimal carrier-phase combinations for modernized triple-frequency GPS. J Geod 82(9):555-564

Collins P, Lahaye F, Héroux P, Bisnath S (2008) Precise point positioning with ambiguity resolution using the decoupled clock model. ION 2008, Savannah, US, pp 1315-1322

Dai L, Eslinger D, Sharpe T (2007) Innovative algorithms to improve long range RTK reliability and availability. ION NTM 2007, San Diego CA, pp 860-872

Deng C, Tang W, Liu J, Shi C (2014) Reliable single-epoch ambiguity resolution for short baselines using combined GPS/BeiDou system. GPS Solut 18(3):375-386

Deo M, El-Mowafy A (2016) Triple-frequency GNSS models for PPP with float ambiguity estimation: performance comparison using GPS. Survey Rev. DOl: https://doi.org/10.1080/00396265.2016.1263179

Dodson AH, Shardlow PJ, Hubbard LCM, Elgered G, Jarlemark POJ (1996) Wet tropospheric effects on precise relative GPS height determination. J Geod 70(4):188-202

Feng Y (2008) GNSS three carrier ambiguity resolution using ionosphere-reduced virtual signals. J Geod 82(12):847-862

Feng Y, Li B (2008) A benefit of multiple carrier GNSS signals: regional scale network-based RTK with doubled inter-station distances. J Spat Sci 53: 135-147

Feng Y, Li B (2010) Wide area real time kinematic decimetre positioning with multiple carrier GNSS signals. Sci China Earth Sci 53(5):731-740

Feng Y, Rizos C (2005) Three carrier approaches for future global, regional and local GNSS positioning services: concepts and performance perspectives. ION GNSS 2005, Long Beach, CA, pp 2277-2287

Fernández-Plazaola U, Martín-Guerrero TM, Entrambasaguas JT (2008) A new method for three-carrier GNSS ambiguity resolution. J Geod 82(4-5):269-278

Fernández-Plazaola U, Martín-Guerrero TM, Entrambasaguas-Muñoz JT, Martín-Neira M (2004) Null meth-od applied to three frequencies. J Geod 78(1-2):96-102

Forssell B, Martín-Neira M, Harris R (1997) Carrier phase ambiguity resolution in GNSS-2. ION GPS 1997, Kansas City, MO, pp 1727-1736

Ge M, Gendt G, Rothacher M, Shi C, Liu J (2008) Resolution of GPS carrier-phase ambiguities in precise point positioning (PPP) with daily observations. J Geod 82(7):389-399

Geng J, Bock Y (2013) Triple-frequency GPS precise point positioning with rapid ambiguity resolution. J Geod 87(5):449-460
Gu S, Lou Y, Shi C, Liu J (2015) BeiDou phase bias estimation and its application in precise point positioning with triple-frequency observable. J Geod 89(10):979-992

Guo F, Zhang X, Wang J (2015) Timing group delay and differential code bias corrections for BeiDou positioning. J Geod 89(5):427-445

Guo F, Zhang X, Wang J, Ren X (2016) Modeling and assessment of triplefrequency BDS precise point positioning. J Geod 90:1223-1235

Hatch R, Jung J, Enge P (2000) Civilian GPS: the benefits of three frequencies. GPS Solut 3(4):1-9

He H, Li J, Yang Y, Xu J, Guo H, Wang A (2014) Performance assessment of single- and dual-frequency BeiDou/GPS single-epoch kinematic positioning. GPS Solut 18(3):393-403

Henkel P, Günther C (2012) Reliable integer ambiguity resolution: multi-frequency code carrier linear combinations and statistical a priori knowledge of attitude. J Inst Nav 59(1):61-75

Laurichesse D, Mercier F, Berthias J, Bijac J (2008) Real time zero-difference ambiguities fixing and absolute RTK. ION 2008, Savannah, US, pp 747-755

Leick A (2004) GPS satellite surveying, 3rd edn. John Wiley, New York

Li B (2016) Stochastic modeling of triple-frequency BeiDou signals: estimation, assessment and impact analysis. J Geod 90:593-610

Li B, Feng Y, Gao W, Li Z (2015a) Real-time kinematic positioning over long baselines using triple-frequency BeiDou signals. IEEE Trans Aerosp Electron Syst 51(4):1-16

Li B, Feng Y, Shen Y (2010a) Three carrier ambiguity resolution: distanceindependent performance demonstrated using semi-generated triple frequency GPS signals. GPS Solut 14(2):177-184

Li B, Feng Y, Shen Y, Wang C (2010b) Geometry-specified troposphere decorrelation for subcentimeter real-time kinematic solutions over long baselines. J Geophys Res 115:B11404

Li B, Li Z, Zhang Z, Tan Y (2017a) ERTK: extra-wide-lane RTK of triple-frequency GNSS signals. J Geod 91:1031-1047

Li B, Zhang L, Verhagen S (2017b) Impacts of BeiDou stochastic model on reliability: overall test, w-test and minimal detectable bias. GPS Solut 21: 1095-1112.

Li B, Shen Y, Feng Y, Gao W, Yang L (2014a) GNSS ambiguity resolution with controllable failure rate for long baseline network RTK. J Geod 88(2):99-112

Li B, Shen Y, Lou L (2011a) Efficient estimation of variance and covariance components: a case study for GPS stochastic model evaluation. IEEE Trans Geos Remote Sens 49(1):203-210

Li B, Shen Y, Xu P (2008) Assessment of stochastic models for GPS measurements with different types of receivers. Chi Sci Bull 53(20):3219-3225

Li B, Shen Y, Zhang X (2013) Three frequency GNSS navigation prospect demonstrated with semi-simulated data. Adv Space Res 51(7):1175-1185

Li B, Teunissen PJG (2014) GNSS antenna array-aided CORS ambiguity resolution. J Geod 88(4):363-376

Li B, Verhagen S, Teunissen PJG (2014b) Robustness of GNSS integer ambiguity resolution in the presence of atmospheric biases. GPS Solut 18(2):283-296

Li H, Li B, Xiao G, Wang J, Xu T (2016) Improved method for estimating the interfrequency satellite clock bias of triple-frequency GPS. GPS Solut 20:751-760

Li T, Chen Q, Wang J (2015b) Enhanced RTK integer ambiguity resolution with BeiDou triple-frequency observations, China Satellite Navigation Conference (CSNC) 2015, vol III. Springer Berlin Heidelberg, pp 227-238

Li T, Wang J, Laurichesse D (2014) Modeling and quality control for reliable precise point positioning integer ambiguity resolution with GNSS modernization. GPS Solut 18(3):429-442

Li X, Zhang X, Ge M (2011b) Regional reference network augmented precise point positioning for instantaneous ambiguity resolution. J Geod 85(3):151-158

Montenbruck O, Hauschild A, Steigenberger P, Hugen-tobler U, Teunissen P, Nakamura S (2013) Initial assessment of the COMPASS/BeiDou-2 regional navigation satellite system. GPS Solut 17(2):211-222

Odolinski R, Teunissen PJG, Odijk D (2013) An analysis of combined COMPASS/ BeiDou-2 and GPS single- and multi-frequency RTK positioning. ION PNT 2013, Honolulu, Hawaii, pp 69-90

Richert T, El-Sheimy N (2007) Optimal linear combinations of triple frequency carrier phase data from future global navigation satellite systems. GPS Solut 11(1):11-19

Shi C, Zhao Q, Hu Z, Liu J (2013) Precise relative positioning using real tracking data from COMPASS GEO and IGSO satellites. GPS Solut 17(1):103-119

Teunissen P, Joosten P, Tiberius C (2002) A comparison of TCAR, CIR and LAMBDA GNSS ambiguity resolution. ION GPS 2002, Portland, OR, pp 2799-2808

Teunissen PJG (1995) The least-squares ambiguity decorrelation adjustment: a method for fast GPS integer ambiguity estimation. J Geod 70:65-82

Teunissen PJG (2001) Integer estimation in the presence of biases. J Geod 75(7-8):399-407 
Tiberius C, Kenselaar F (2000) Estimation of the stochastic model for GPS code and phase observation. Surv Rev 35(277):441-454

Verhagen S, Tiberius C, Li B, Teunissen PJG (2012) Challenges in ambiguity resolution: Biases, weak models, and dimensional curse. NAVITEC, 2012 6th ESA Workshop on, Noordwijk, Netherlands, pp 1-8

Vollath U, Birnbach S, Landau H (1998) Analysis of three carrier ambiguity resolution (TCAR) technique for precise relative positioning in GNSS-2. ION GPS 1998:417-426

Wang J, Stewart M, Sakiri M (1998) Stochastic modeling for static GPS baseline data processing. J Surv Engi 124(4):171-181

Wang K, Rothacher M (2013) Ambiguity resolution for triple-frequency geometryfree and ionosphere-free combination tested with real data. J Geod 87(6): $539-553$

\section{Submit your manuscript to a SpringerOpen ${ }^{\mathcal{D}}$} journal and benefit from:

- Convenient online submission

- Rigorous peer review

- Open access: articles freely available online

- High visibility within the field

- Retaining the copyright to your article

Submit your next manuscript at $>$ springeropen.com 